\title{
THE EUCLIDEAN SYMMETRIC ISOSCELES QUEASY FOUR-POINT PROPERTIES
}

\author{
J. E. VALENTINE AND S. G. WAYMENT
}

\begin{abstract}
The purpose of this paper is to show that a complete, convex, externally convex metric space is generalized euclidean if and only if it has the euclidean symmetric isosceles queasy fourpoint property or the euclidean external isosceles queasy fourpoint property.
\end{abstract}

1. Introduction. Since Wilson [10] characterized generalized euclidean spaces among the class of complete, convex, externally convex metric spaces as those which possess the euclidean four-point property, Blumenthal ([1], [2]) and others have introduced weaker euclidean four-point properties which yield the characterization. Freese [7] introduced an external isosceles feeble euclidean four-point property and proved that it yields a characterization of generalized euclidean space. In [7] Freese asked if an isosceles feeble euclidean four-point property would also provide a characterization. Valentine [9] gave an affirmative answer to his question. Day [5] introduced the euclidean queasy four-point property and showed that it characterizes generalized euclidean space. The purpose of this paper is to consider the symmetric isosceles queasy and external symmetric isosceles queasy four-point properties and show that a complete, convex, externally convex metric space is generalized euclidean if and only if it possesses one of these isosceles four-point properties. It then follows that Valentine's and Freese's characterizations ([9], [7]) are special cases of this work.

2. The four-point properties. The following classes of metric quadruples have been introduced by Wilson, Blumenthal and others.

A metric quadruple $p_{1}, p_{2}, p_{3}, p_{4}$ belongs to class

$\mathrm{C}_{0}$ if and only if $p_{1}, p_{2}, p_{3}, p_{4}$ are pairwise distinct,

$\mathrm{C}_{1}$ if and only if $p_{1}, p_{2}, p_{3}, p_{4}$ are pairwise distinct and it contains a linear triple,

Received by the editors November 24, 1970.

AMS 1969 subject classifications. Primary 5250.

Key words and phrases. Convex, externally convex, euclidean four-point property, euclidean feeble four-point property, euclidean isosceles feeble four-point property, euclidean external isosceles feeble four-point property, euclidean symmetric isosceles queasy and external isosceles queasy four-point properties, euclidean queasy fourpoint property.

(c) American Mathematical Society 1972 
$\mathrm{C}_{2}$ if and only if $p_{1}, p_{2}, p_{3}, p_{4}$ are pairwise distinct, $p_{3}$ is between $p_{2}, p_{4}$ and $p_{2} p_{3}=p_{3} p_{4}$,

$\mathrm{C}_{3, k}$ if and only if $k>1$ and $p_{1}, p_{2}, p_{3}, p_{4}$ are pairwise distinct, $p_{2}$ is between $p_{1}$ and $p_{3}$, while $p_{4} p_{1}=p_{4} p_{2}$ and $k \cdot p_{1} p_{2}=p_{1} p_{3}$,

$\mathrm{C}_{4, k}$ if and only if $0<k<1$ and $p_{1}, p_{2}, p_{3}, p_{4}$ are pairwise distinct, $p_{3}$ is between $p_{1}$ and $p_{2}$, while $p_{4} p_{1}=p_{4} p_{2}$ and $k \cdot p_{1} p_{2}=p_{1} p_{3}$.

Definitions. A metric space has the euclidean, euclidean weak, euclidean feeble, euclidean $k$-external isosceles feeble, euclidean $k$ isosceles feeble four-point property provided every quadruple of its points of class $\mathrm{C}_{0}, \mathrm{C}_{1}, \mathrm{C}_{2}, \mathrm{C}_{3, k}, \mathrm{C}_{4, k}$, respectively, is congruently embeddable in euclidean space. A metric space is said to have the euclidean queasy four-point property provided for each two of its points $p$ and $r$ there exists a point $q$ between them such that for all points $s$ of the space the quadruple $p, q, r, s$ is congruently embeddable in euclidean space. A metric space is said to have the euclidean symmetric isosceles queasy four-point property provided for each pair of its distinct points $p$ and $r$ there is a number $\lambda=\lambda(p, r)(0<\lambda<1)$ such that if $q$ is a point between $p$ and $r$ such that $p q=\lambda p r$ or $q r=\lambda p r$, then for each point $s$ in the space for which $p s=r s$ the quadruple $p, q, r, s$ is congruently embeddable in euclidean space.

A metric space is said to have the euclidean external symmetric isosceles queasy four-point property provided for each pair of its distinct points $p$ and $r$ there is a number $\lambda=\lambda(p, r)(1<\lambda<k, k$ arbitrary but fixed $)$ such that if $q$ is a point for which $r$ is between $p$ and $q$ and $p q=\lambda p r$ or $p$ is between $q$ and $r$ and $q r=\lambda p r$, then for each point $s$ in the space for which $p s=r s$ the quadruple $p, q, r, s$ is congruently embeddable in euclidean space.

3. The euclidean symmetric isosceles queasy four-point property. From [9] it is known that the euclidean isosceles feeble four-point property (with $k=\frac{1}{2}$ ) characterizes generalized euclidean space among the class of complete, convex, externally convex metric spaces. In order to show the euclidean symmetric isosceles queasy four-point property effects such a characterization it suffices to show that a metric space possessing this property also possesses the euclidean isosceles feeble four-point property $\left(k=\frac{1}{2}\right)$. In the discussion that follows, $M$ will denote a complete, convex, externally convex metric space that possesses the euclidean symmetric isosceles queasy four-point property.

LeMma 3.1. Each two distinct points of $M$ are endpoints of exactly one metric segment.

Proof. Since $M$ is complete and convex, each two points of $M$ are endpoints of at least one segment. Assume for an indirect proof that $M$ 
contains points $p_{1}$ and $p_{3}$ and distinct points $p_{4}$ and $p_{4}^{*}$ which are midpoints of $p_{1}$ and $p_{3}$. By the euclidean symmetric isosceles queasy four-point property there is a point $p_{2}$ between $p_{1}$ and $p_{3}$ such that each of the quadruples $p_{1}, p_{2}, p_{3}, p_{4}$ and $p_{1}, p_{2}, p_{3}, p_{4}^{*}$ is congruently embeddable in euclidean space. Since $p_{1}, p_{2}, p_{3} ; p_{1}, p_{3}, p_{4}$; and $p_{1}, p_{3}, p_{4}^{*}$ are linear triples, by the two-triple property for euclidean space, it follows that $p_{2}, p_{4}^{*}, p_{3}$; $p_{2}, p_{4}, p_{3} ; p_{1}, p_{2}, p_{4}^{*} ;$ and $p_{1}, p_{2}, p_{4}$ are also linear triples. Since $p_{4}$ and $p_{4}^{*}$ are midpoints of $p_{1}$ and $p_{3}$, it is easily seen that $p_{2} p_{4}=p_{2} p_{4}^{*}$ and no generality is lost in assuming $p_{2} p_{4} p_{3}$ holds. The euclidean symmetric isosceles queasy four-point property implies the existence of a point $p$ between $p_{4}$ and $p_{4}^{*}$ such that the quadruples $p_{2}, p_{4}, p_{4}^{*}, p$ and $p_{3}, p_{4}, p_{4}^{*}, p$ are congruently embeddable in euclidean space and consequently $p p_{2}<p_{2} p_{4}$ and $p p_{3}<p_{3} p_{4}$ so $p p_{2}+p p_{3}<p_{2} p_{4}+p_{4} p_{3}=p_{2} p_{3}$, contrary to the triangle inequality. Thus for each pair of distinct points of $M, M$ contains exactly one midpoint and hence each pair of distinct points lie on a unique metric segment.

\section{Lemma 3.2. Metric segments in $M$ admit unique prolongations.}

Proof. Since $M$ is externally convex, the segment $S(p, q)$ admits a prolongation. Suppose $S(p, q)$ has two prolongations through $q$. Let $s$ be a point on one prolongation and $t$ a point on the other prolongation such that $q p=q s=q t$. Let $r$ denote a point of $S(s, t)$ for which the quadruples $p, s, r, t$ and $q, s, r, t$ are embeddable in the euclidean plane. Applying the law of cosines to the triples $p, s, t$ and $p, s, r$ to obtain $\cos \Varangle s: t, p$ we obtain

$$
\left(p s^{2}+s t^{2}-p t^{2}\right) / 2 \cdot p s \cdot s t=\left(p s^{2}+r s^{2}-p r^{2}\right) / 2 \cdot p s \cdot r s .
$$

In a similar manner we obtain $\cos \Varangle s: t, q$ in two ways and we have

$$
\left(q s^{2}+s t^{2}-q t^{2}\right) / 2 q s \cdot s t=\left(q s^{2}+r s^{2}-q \dot{r}^{2}\right) / 2 q s \cdot r s .
$$

If $\lambda \cdot s t$ is substituted for $r s$ in (1) and (2) and the resulting equations are solved for $\lambda \cdot s t^{2}$ it follows that

$$
3 q s^{2}=p r^{2}-q r^{2}
$$

However, by the triangle inequality $p r \leqq q s+q r$ and consequently $3 q s^{2}=p r^{2}-q r^{2}<q s^{2}+2 \cdot q s \cdot q r<q s^{2}+2 q s^{2}=3 q s^{2}$, since $q, r, s, t$ are congruently embeddable in the euclidean plane implies $q r<q s$. This contradiction completes the proof.

The results of Lemmas 3.1 and 3.2 may now be combined to obtain

LEMMA 3.3. Each two distinct points of $M$ are contained in exactly one line of $M$. 
Lemma 3.4. The space $M$ possesses the euclidean isosceles feeble fourpoint property $\left(k=\frac{1}{2}\right)$.

Proof. Suppose $p, q, r$ are points of $M$ such that $p q=p r$, and suppose $m$ is the midpoint of $q$ and $r$. By the euclidean symmetric isosceles queasy four-point property $M$ contains a point $q_{1}$ between $q$ and $r$ such that $\lambda q r=q q_{1}$ and $p, q, q_{1}, r$ are congruently embeddable in the euclidean plane. In the event that $\lambda=\frac{1}{2}$ the proof is complete. If $\lambda \neq \frac{1}{2}$, then as above a point $r_{1}$ exists such that $\lambda q r=r r_{1}$ and $p, q, r_{1}, r$ are congruently embeddable in the euclidean plane. Since $q q_{1}=r r_{1}$, it follows from the euclidean law of cosines that $p q_{1}=p r_{1}$. Let $K(q, r)=\{\lambda \in(0,1) \mid$ there exist points $x$ in $S(q, m), y$ in $S(m, r)$ such that $q x / q r=r y / q r=\lambda$ and $p x=p y\}$. If $\lambda(q, r) \neq \frac{1}{2}$, then $K(q, r)$ is a nonempty subset of real numbers bounded above by $\frac{1}{2}$ and hence lub $K(q, r)$ exists and is less that or equal to $\frac{1}{2}$. If $\operatorname{lub} K(q, r)=\frac{1}{2}$, then for each $n, S(q, r)$ contains points $q_{n}, r_{n}$ such that $q_{n} m r_{n}$ holds, $q q_{n} / q r=r r_{n} / q r>\frac{1}{2}-1 / n$ and $p q_{n}=p r_{n}$, and so $\lim q_{n}=m$, $\lim r_{n}=m$. It follows from the continuity of the metric that $p, q, r, m$ are congruently embeddable in the euclidean plane. If lub $K(q, r)<\frac{1}{2}$, then $S(q, m), S(m, r)$ contain points $x$ and $y$, respectively, such that the quadruple $p, q, r, x$; $p, q, r, y ; p, x, y, m$ are congruently embeddable in the euclidean plane and $p x=p y, q x=r y$. Applications of the euclidean law of cosines yields $p, q, r, m$ are congruently embeddable in the euclidean plane.

THEOREM 1. A complete, convex, externally convex metrix space is generalized euclidean if and only if it possesses the euclidean symmetric isosceles queasy four-point property.

Proof. By Lemma 3.4 the euclidean symmetric isosceles queasy fourpoint property is equivalent to the euclidean isosceles feeble four-point property $\left(k=\frac{1}{2}\right)$. Therefore, by a result of [9], the conclusion follows.

Clearly the euclidean $k$-isosceles feeble four-point property implies the euclidean symmetric isosceles queasy four-point property and we thus have

THEOREM 2. A complete, convex, externally convex metric space is generalized euclidean if and only if it possesses the euclidean $k$-isosceles feeble four-point property.

It should be noted that it follows as in [5] that if a complete, externally convex, metric space possesses the euclidean symmetric isosceles queasy (feeble) four-point property and contains for each pair of its distinct points $q$ and $r$ a point $p$ such that $p q=p r$, then the metric space is convex.

4. The euclidean external symmetric isosceles queasy four-point property. As was noted in the introduction, Freese [7] characterized generalized 
euclidean space using the euclidean $k$-external isosceles feeble four-point property with $k=\frac{3}{2}$. A careful examination of the proofs of his lemmas shows that only minor changes need to be made to obtain the results using the euclidean external symmetric isosceles queasy four-point property. We thus note the following two theorems.

THEOREM 3. A complete, convex, externally convex metric space is generalized euclidean if and only if it possesses the euclidean external symmetric isosceles queasy four-point property.

THEOREM 4. A complete, convex, externally convex metric space is generalized euclidean if and only if it possesses the euclidean $k$-external isosceles feeble four-point property.

As in [5] the euclidean external symmetric isosceles queasy and the euclidean $k$-external isosceles feeble four-point properties imply the space is externally convex provided for each pair of distinct points $q, r$ there is a point $p$ such that $p q=p r$.

5. Concluding remark. The euclidean isosceles queasy four-point property may be stated as follows.

A metric space $M$ possesses the euclidean isosceles queasy four-point property provided for each pair of its distinct points $p, r$ there is a point $q$ between $p$ and $r$ such that for each point $s$ of $M$ whenever $p s=r s$, the quadruple $p, q, r, s$ is congruently embeddable in euclidean space.

It should be noted that the difference between the symmetric queasy and the queasy four-point properties is that with the exception when $\lambda=\frac{1}{2}$ the symmetric queasy insures the existence of at least two points $q, q^{\prime}$ between distinct points $p$ and $r$ such that for each $s$ of the space, $p s=r s$ implies the quadruples $p, q, r, s$ and $p, q^{\prime}, r, s$ are embeddable in the euclidean plane. In this work we only used the stronger form in the proof of Lemma 3.4. It would be interesting to know if the euclidean isosceles queasy four-point property effects such a characterization. If it does, this would not only be a strengthening of our result, but it would also strengthen the result of Day [5]. Similarly it would be interesting to know if the symmetric property of the euclidean external isosceles queasy four-point property could be deleted. This property is not needed until Freese's Lemma 6.

\section{REFERENCES}

1. L. M. Blumenthal, Theory and applications of distance geometry, Clarendon Press, Oxford, 1953. MR 14, 1009.

2. - An extension of a theorem of Jordon and von Neumann, Pacific J. Math. 5 (1955), 161-167. MR 16, 1139.

3. L. M. Blumenthal and E. Z. Andalafte, Metric characterizations of Banach and Euclidean spaces, Fund. Math. 55 (1964), 23-55. MR 29 \#2625. 
4. M. M. Day, Some characterizations of inner-product spaces, Trans. Amer. Math. Soc. 62 (1947), 320-337. MR 9, 192.

5. - On criteria of Kasahara and Blumenthal for inner-product spaces, Proc. Amer. Math. Soc. 10 (1959), 92-100. MR 21 \#5140.

6. M. Fréchet, Sur la definition axiomatique d'une classe d'espaces vectoriels distances applicables vectorellement sur l'espace de Hilbert, Ann. of Math. (2) 36 (1935), 705-718.

7. R. W. Freese, Criteria for inner product spaces, Proc. Amer. Math. Soc. 19 (1968), 953-958. MR 37 \#3452.

8. P. Jordon and J. von Neumann, On inner-products in Linear metric spaces, Ann. of Math. (2) 36 (1935), 719-723.

9. J. E. Valentine, On criteria of Blumenthal for inner-product spaces, Fund. Math. 72 (1971), 265-269.

10. W. A. Wilson, A relation between metric and Euclidean spaces, Amer. J. Math. 54 (1932), 505-517.

Department of Mathematics, Utah State University, Logan, Utah 84321 